\title{
Variación mensual y anual de la riqueza y abundancia de aves en un mosaico agrícola periurbano tropical
}

\author{
Luis Sandoval \\ Escuela de Biología, Universidad de Costa Rica, San Pedro, Montes de Oca, Costa Rica, CP-11501-2060; \\ biosandoval@hotmail.com
}

Recibido 12-X-2017. Corregido 18-XII-2018. Aceptado 17-I-2019.

\begin{abstract}
Annual and monthly variation on bird species richness and abundance inside a tropical semiurban agricultural landscape. Species richness inside agricultural landscape has been studied intensively and according to the type of landscape structure. However, the species phenology inside the mostly agricultural landscape is poorly understood. Therefore, my objective was to analyze species richness and individual abundance changes between months inside a semiurban agricultural landscape. Additionally, I analyzed if changes in species richness varied with the trophic guild and forest dependence of each species. From 2002 to 2010I I sampled 30 ha of coffee plantations and cattle land with natural successional regenerations and rural development. I recorded every individual listened and seen along a $3 \mathrm{~km}$ long transect once a month. The species richness changed between months and was higher between January and March (dry season in the study area) for all residence status (i.e., residents and north, south, and altitudinal migrants). Small frugivore species richness was higher between January and February, insectivore species richness was higher in September. The other food guilds had steady richness and abundance throughout the year. Species with a medium forest dependence showed higher species richness between January and June. Species richness phenology changed along the year depending on residence status, trophic guild, and forest dependence, a finding that should be taken into account in future conservation of this type of habitat.
\end{abstract}

Key words: bird abundance; bird richness; cattle land; coffee plantations; Costa Rica; landscape structure; species phenology.

Sandoval, L. (2019). Variación mensual y anual de la riqueza y abundancia de aves en un mosaico agrícola periurbano tropical. Revista de Biología Tropical, 67(2) Suplemento, S298-S314.

La expansión agrícola especialmente en los trópicos, afecta la riqueza y abundancia de plantas y animales de los sitios donde ocurre y sus alrededores (Foley et al., 2005; Foley et al., 2011), debido a la modificación de la cobertura natural y su remplazo por coberturas más homogéneas como pastizales o monocultivos (Ramankutty, Evan, Monfreda, \& Foley, 2008). Por lo tanto, comprender como estos cambios afectan la abundancia y riqueza de las especies nativas, es imperativo si queremos ayudar a que las especies nativas permanezcan y utilicen estos paisajes modificados (Fischer et al., 2008; Vilchez Mendoza et al., 2014).
No todas las áreas agrícolas tienden a ser plantaciones extensivas y monocultivistas; pues en las regiones rurales cercanas a las ciudades existen pequeños desarrollos agrícolas que presentan una matriz más heterogénea de hábitats (Perfecto, Rice, Greenberg, \& Van der Voort, 1996; Miles \& Kapos, 2008). Por ejemplo, una mezcla de diferentes tipos de plantaciones (ej.: café, cítricos, pastos, caña de azúcar, o forestales), con vegetación natural remanente y regeneraciones naturales, cercas vivas y pequeños desarrollos urbano-rurales (Weibull, Bengtsson, \& Nohlgren, 2000; Tscharntke et al., 2008). Esta heterogeneidad ambiental podría 
incrementar la complejidad de la composición y estructura de las comunidades de animales, al mantener características del hábitat previo, que pueden favorecer a especies nativas, así como crear nuevas condiciones de hábitat atractivas para especies colonizadoras (Wunderle \& Latta, 1996; Greenberg, Bichier, \& Sterling, 1997; Greenberg, Bichier, Cruz Agnon, \& Reitsma, 1997).

Por el contrario, estos cambios en el uso de suelo y la ocurrencia de una mayor heterogeneidad ambiental también podrían influir negativamente en la abundancia de las especies nativas y positivamente en la abundancia de las especies colonizadoras (Stiles, 1990; Biamonte, Sandoval, Chacón, \& Barrantes, 2011; Barrantes, Ocampo, Ramírez-Fernández, \& Fuchs, 2016). Además, la reducción y fragmentación del hábitat natural puede reducir el tamaño de las poblaciones nativas, al incrementar el aislamiento entre poblaciones, lo cual limita el intercambio genético interpoblacional (Bierregaard \& Lovejoy, 1988; Hughes, Daily, \& Ehrlich, 1997; Manhães \& Loures-Ribeiro, 2005). Otra consecuencia de la reducción del hábitat nativo es el aumento inicial de la densidad de las poblaciones, acelerando la transmisión de enfermedades o incrementando el número de interacciones agresivas entre los individuos (Costantini, Greives, Hau, \& Partecke, 2014; Soulsbury \& White, 2016). Por otro lado, la fragmentación aumenta el área de ocurrencia y promueve el flujo genético dentro de especies de zonas abiertas o con disturbios, al conectar poblaciones previamente aisladas (Meillère, Brischoux, Parenteau, \& Angelier, 2015; Beninde et al., 2016).

Uno de los grupos taxonómicos donde la mayoría de especies dependientes de hábitats naturales se ven afectados por estos cambios de uso de suelo debido a la agricultura son las aves, ya que muchas especies están restringidas a un tipo particular de hábitat y si ese hábitat disminuye o se elimina, lo hacen las especies asociadas (Stiles, 1985; Stotz, Fitzpatrick, Parker, \& Moskovits, 1996). Por esta razón la riqueza y abundancia de aves se han utilizado como un indicador del efecto producido por el cambio de uso de suelo, porque permiten comparar las comunidades de aves entre sitios con diferentes tipos de cobertura agrícola y natural (Tilghman, 1987; Tscharntke et al., 2008). No obstante, la mayoría de estudios previos buscaron relacionar la matriz agrícola con la riqueza de especies y su abundancia, pero los cambios en la riqueza y abundancia a través del año y entre años, han sido escasamente evaluados. Por lo tanto mi objetivo fue analizar la variación anual en la riqueza y abundancia de las aves que ocurre en un mosaico agrícola-rural a lo largo de un periodo de ocho años. Adicionalmente analicé los cambios en riqueza y abundancia de las especies tomando en cuenta el grado de dependencia por los hábitats (ej.: bosque o zonas abiertas), la dieta, y si son o no residentes en el país.

\section{MATERIALES Y MÉTODOS}

Área de estudio y muestreo: El estudio lo realicé en Getsemaní, provincia de Heredia, Costa Rica $\left(10^{\circ} 01^{\prime} 43\right.$ ” N, 8406’44” W, 1300 msnm), En un área de aproximadamente 30 ha, donde: $40 \%$ corresponde a cafetales (con sombra y sin sombra con cercas vivas), $40 \%$ a pastizales para ganadería (con árboles aislados y cercas vivas), $10 \%$ de vegetación en regeneración natural (incluyendo vegetación riparia), y $10 \%$ de zonas urbano-rural (Fig. 1; Sandoval, 2011). Durante el periodo de estudio (diciembre 2002 a abril 2010), no se dio ningún cambio en el uso de suelo, salvo pequeñas podas de cafetales debido al mantenimiento, caída azarosa de árboles o arbustos debido a las lluvias o vientos fuertes, o muerte de plantas por su senescencia normal.

Para recolectar lo datos recorrí a velocidad moderada en promedio una vez al mes (2002 solo un muestreo y 2010 solo tres muestreos) un transecto de $3 \mathrm{~km}$ de longitud con bandas fijas de $25 \mathrm{~m}$ a cada lado, desde las 06:00 hasta las 09:00, que abarcaba la mayoría del área de estudio y pasaba por los cuatro tipos de uso de suelo del área (potreros, cafetales, regeneración natural y urbano rural; Fig. 1). Para evitar recontar individuos en sitios diferentes el punto 


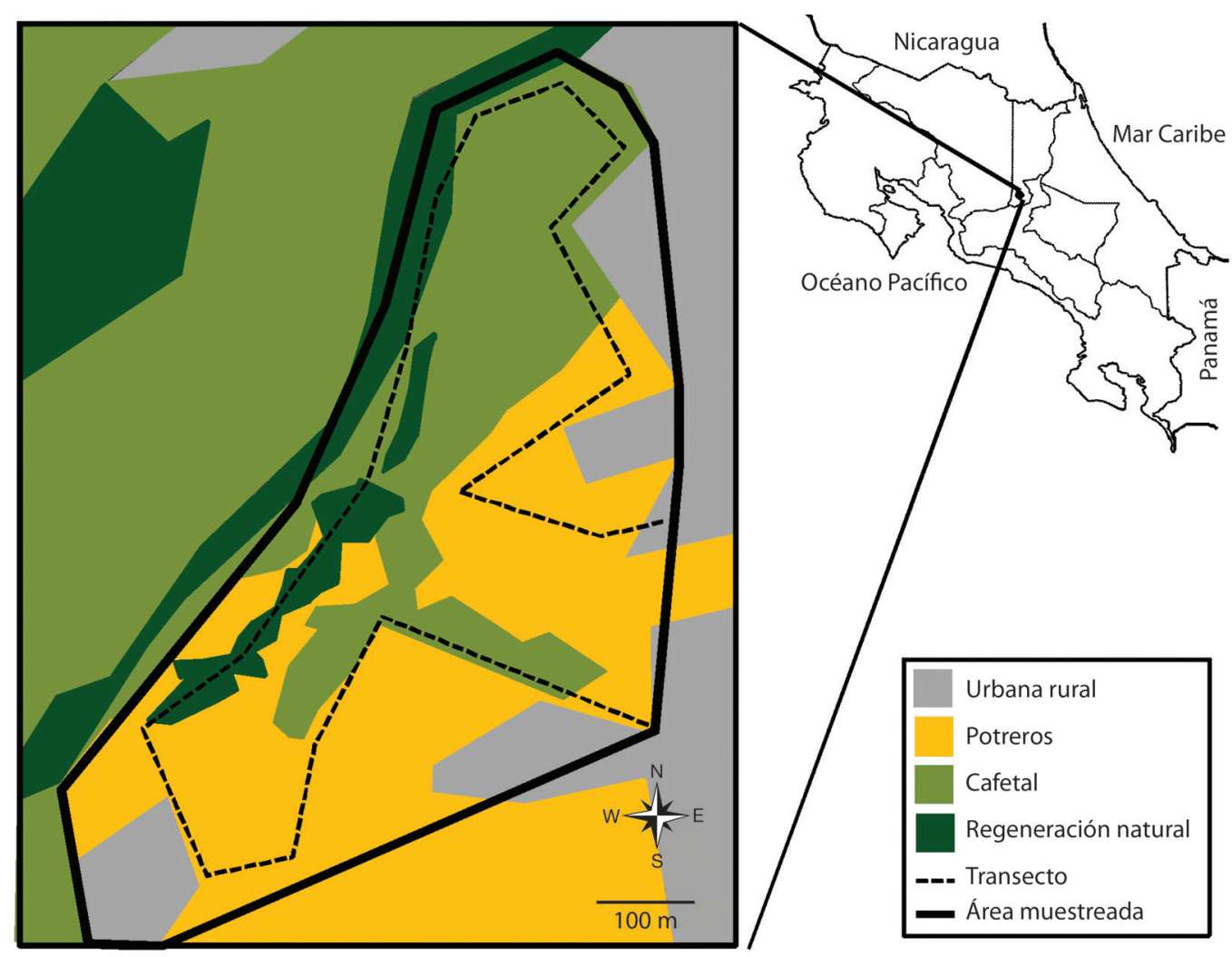

Fig. 1. Área de estudio (Getsemaní, provincia de Heredia, Costa Rica) mostrando los cuatro usos de suelos principales y el transecto utilizado para muestrear las aves de 2002 a 2010. Mapa de uso de suelo creado con base en imágenes de Google Earth durante el periodo de estudio. En la esquina superior derecha el mapa de Costa Rica indicando la ubicación del sitio de estudio en el país.

Fig. 1. Four main land uses at the study area (Getsemaní, Heredia province, Costa Rica) with transect used on bird sample from 2002 to 2010. Land use was created using Google Earth images form study period. In the upper right corner is the Costa Rica map showing the location in the country.

más cercano entre dos sectores del transecto fueron $100 \mathrm{~m}$. Además, alterné la dirección de inicio del muestreo entre conteos para aumentar la probabilidad de detección de los individuos en todos los hábitats y áreas de muestreo.

Durante el recorrido, anoté la cantidad de individuos observados y escuchados por especie. Siguiendo la dirección de desplazamiento de las aves o bandadas, e intentando dentro de lo posible estimar si se dirigían a sectores aun no muestreados del transecto, evité contar los individuos de esas especies si se encontraban a menos $\leq 100 \mathrm{~m}$ de la primera observación para no sobreestimar la abundancia de individuos por especie. En especies que cazan y pasan la mayor parte del tiempo volando (ej.: golondrinas o zopilotes) anote el número máximo de individuos observados en cualquier momento del conteo, en lugar de ir adicionando los individuos observados (ej.: si observaba dos golondrinas al iniciar el conteo, cinco a la mitad, y dos al final el número de golondrinas anotado sería cinco, y no nueve) para evitar sobreestimaciones.

Todas las especies fueron clasificadas según su residencia en el país: residentes, si permanecen todo el año en la zona; migratorios altitudinales residentes, si tiene desplazamientos a lo largo de las vertientes de Costa Rica; migratorios norteños, si se reproducen en 
Norte América y pasan el invierno del hemisferio norte en Costa Rica; y migratorios sureños, si se reproducen en Costa Rica y migran hacia Sur América después de reproducirse (Stiles \& Skutch, 1989). Además, clasifiqué las especies según el grado de dependencia del bosque siguiendo lo propuesto por Stiles (1985), y modificado por Sandoval \& Barrantes (2009) y Biamonte et al. (2011): $1=$ especies que necesitan bosques maduros para sobrevivir, 2 $=$ especies que viven en áreas con al menos $50 \%$ de cobertura boscosa, $3=$ especies que viven en áreas abiertas; 4 = especies que viven en lagunas o humedales, y aquellas especies con valores 1-2 y 2-3 en Stiles (1985) fueron reclasificados como 1.5 y 2.5 respectivamente. Donde especies con valor de 1.5 son especies que viven tanto en bosques maduros y áreas con al menos $50 \%$ de cobertura boscosa; y 2.5 son especies que viven tanto en áreas con al menos $50 \%$ de cobertura boscosa y áreas abiertas.

Finalmente, según los criterios de Stiles y Skutch (1989) se clasificaron como: cazadores, si se alimentan de vertebrados o carroña; frugívoros grandes, si pesan $>20 \mathrm{~g}$ y se alimentan de frutas; frugívoros pequeños, si pesan $\leq 20$ g y se alimentan de frutas; insectívoros, si se alimenta de insectos u artrópodos; nectarivoros, si se alimentan de néctar; semilleros, si se alimentan de semillas; y omnívoros, si su dieta está compuesta por dos o más tipos de alimentos en proporción similar.

Análisis estadísticos: Realicé un modelo lineal mixto (MLM; Neter, Kutner, Nachtsheim, \& Wasserman, 1996) por categoría de residencia en el país para comparar como cambia la riqueza de especies (variable dependiente) entre años (variable independiente) y utilizando como variable aleatoria el mes. Para las categorías de residencia: todos (que incluye todas las especies), solo residentes y migratorios altitudinales, no utilicé los datos de 2002 (con muestreo únicamente en diciembre) ni del 2010 (con muestreos de enero a marzo). Sin embargo, para la categoría de migratorios norteños si utilicé los datos de todo los años, porque los periodos migratorios empiezan en agosto y terminan en mayo, siendo la variable independiente el periodo migratorio (ej.: 20022003, 2003-2004, y así sucesivamente hasta 2009-2010). Luego utilice otro MLM por categoría de residencia en el país para comparar como cambia la riqueza de especies (variable dependiente) entre meses (variable independiente) y utilizando como variable aleatoria el año. Para comparar si la abundancia y riqueza de todas las especies y las especies residentes por mes fue similar entre meses realicé un análisis de similitud (ANOSIM). En este caso usé como algoritmo de separación de los grupos la distancia de similitud del índice de Morisita, comparando la abundancia y riqueza de todas las especies y de las especies residentes por mes. Para dicho análisis, la variable de agrupación fue cada mes, por lo tanto un resultado significativo indica que al menos hay un mes donde la similitud de los muestreos dentro del mes en términos de la riqueza de especies y abundancia de cada especie fue mayor, que entre los muestreos de diferentes meses.

Finalmente, realicé un análisis lineal generalizados (ALG; con una distribución de Poisson) para cada tipo de dieta y cada grado de dependencia del bosque (agrupe las categorías 1 y 1.5 en una sola para este análisis, debido a que registré muy pocas especies) para comparar si la riqueza de especies (variable dependiente) cambia entre los meses (variable independiente). Para todos los MLM y los ALG que resultaron estadísticamente significativos realicé pruebas post hoc para determinar entre cuales de las variables independientes se encontraban las diferencias, y estas diferencias se mencionan en el texto. Para realizar los MLM y ALG utilicé el software JMP versión 7.0 (SAS Institute, Cary, NC, EEUU), y para los análisis de ANOSIM el software PAST versión 3.21 (Hammer, Harper, \& Ryan, 2001).

\section{RESULTADOS}

De 2002 a 2010 realicé un total de 89 muestreos en los cuales registré un total de 160 especies de aves en el área de estudio (Cuadro 1): 59 migratorias (55 norteñas y 
CUADRO 1

Lista de aves muestreadas de 2002 a 2010 en Getsemaní, Heredia, Costa Rica, a lo largo de un transecto lineal de $3 \mathrm{~km}$, dentro de una matriz agrícola rural de 30 ha

TABLE 1

Bird list at Getsemaní, Heredia, Costa Rica from 2002 to 2010 sampled using $3 \mathrm{~km}$ transect inside 30 ha of agricultural rural matrix

\begin{tabular}{|c|c|c|c|c|}
\hline Taxa & Abundancia & *Residencia & Dependencia del Bosque & Tipo de Dieta \\
\hline \multicolumn{5}{|l|}{ Accipitridae } \\
\hline Buteo albonotatus & 0.06 & $\mathrm{R}$ & 2 & Cazador \\
\hline Buteo brachyurus & 0.07 & $\mathrm{R}$ & 1 & Cazador \\
\hline Buteo plagiatus & 0.36 & $\mathrm{R}$ & 2 & Cazador \\
\hline Buteo platypterus & 2.78 & $\mathrm{MN}$ & 3 & Cazador \\
\hline Buteogallus anthracinus & 0.01 & $\mathrm{R}$ & 2 & Cazador \\
\hline Elanoides forficatus & 0.03 & $\mathrm{R}$ & 2 & Cazador \\
\hline Elanus leucurus & 0.19 & $\mathrm{R}$ & 3 & Cazador \\
\hline \multicolumn{5}{|l|}{ Apodidae } \\
\hline Chaetura pelagica & 0.02 & $\mathrm{MN}$ & 3 & Insectívoro \\
\hline Chaetura vauxi & 0.73 & $\mathrm{R}$ & 3 & Insectívoro \\
\hline Cypseloides niger & 0.79 & $\mathrm{R}$ & 3 & Insectívoro \\
\hline Streptopocne rutilus & 0.02 & $\mathrm{R}$ & 3 & Insectívoro \\
\hline Streptoprocne zonaris & 2.55 & $\mathrm{R}$ & 3 & Insectívoro \\
\hline \multicolumn{5}{|l|}{ Ardeidae } \\
\hline Bubulcus ibis & 2.72 & $\mathrm{R}$ & 3 & Omnívoro \\
\hline \multicolumn{5}{|l|}{ Bombicillidae } \\
\hline Bombycilla cedrorum & 0.69 & $\mathrm{MN}$ & 2.5 & Frugívoro pequeño \\
\hline \multicolumn{5}{|l|}{ Caprimulgidae } \\
\hline Chordeiles minor & 0.01 & $\mathrm{MN}$ & 3 & Insectívoro \\
\hline Nyctidromus albicollis & 0.28 & $\mathrm{R}$ & 3 & Insectívoro \\
\hline \multicolumn{5}{|l|}{ Cardinallidae } \\
\hline Habia rubica & 0.49 & $\mathrm{R}$ & 1.5 & Frugívoro pequeño \\
\hline Passerina cyanea & 0.11 & $\mathrm{MN}$ & 3 & Semillero \\
\hline Pheucticus ludovicianus & 0.36 & $\mathrm{MN}$ & 3 & Frugívoro pequeño \\
\hline Piranga bidentata & 0.37 & $\mathrm{R}$ & 2.5 & Frugívoro pequeño \\
\hline Piranga olivacea & 0.28 & $\mathrm{MN}$ & 3 & Frugívoro pequeño \\
\hline Piranga rubra & 0.48 & $\mathrm{MN}$ & 2.5 & Frugívoro pequeño \\
\hline \multicolumn{5}{|l|}{ Cathartidae } \\
\hline Cathartes aura & 1.29 & $\mathrm{R}$ & 3 & Cazador \\
\hline Coragyps atratus & 2.62 & $\mathrm{R}$ & 3 & Cazador \\
\hline \multicolumn{5}{|l|}{ Charadriidae } \\
\hline Pluvialis dominica & 0.01 & $\mathrm{MN}$ & 4 & Cazador \\
\hline \multicolumn{5}{|l|}{ Ciconiidae } \\
\hline Mycteria americana & 0.03 & $\mathrm{R}$ & 4 & Cazador \\
\hline \multicolumn{5}{|l|}{ Columbidae } \\
\hline Columba livia & 0.96 & $\mathrm{R}$ & 3 & Semillero \\
\hline Columbina inca & 2.65 & $\mathrm{R}$ & 3 & Semillero \\
\hline Columbina passerina & 0.39 & $\mathrm{R}$ & 3 & Semillero \\
\hline Columbina talpacoti & 0.02 & $\mathrm{R}$ & 3 & Semillero \\
\hline Leptotila verreauxi & 8.49 & $\mathrm{R}$ & 2.5 & Semillero \\
\hline Patagioenas fasciata & 0.21 & MA & 2 & Semillero \\
\hline
\end{tabular}


CUADRO 1 (Continuación) / TABLE 1 (Continued)

\begin{tabular}{|c|c|c|c|c|}
\hline Taxa & Abundancia & *Residencia & Dependencia del Bosque & Tipo de Dieta \\
\hline Patagioenas flavirostris & 3.53 & $\mathrm{R}$ & 3 & Semillero \\
\hline Zenaida asiatica & 3.75 & $\mathrm{R}$ & 3 & Semillero \\
\hline \multicolumn{5}{|l|}{ Corvidae } \\
\hline Psilorhinus morio & 5.39 & $\mathrm{R}$ & 3 & Frugívoro grande \\
\hline \multicolumn{5}{|l|}{ Cuculidae } \\
\hline Coccyzus americanus & 0.01 & $\mathrm{MN}$ & 3 & Insectívoro \\
\hline Crotophaga sulcirostris & 3.37 & $\mathrm{R}$ & 3 & Insectívoro \\
\hline Piaya cayana & 1.61 & $\mathrm{R}$ & 2.5 & Insectívoro \\
\hline Tapera naevia & 0.02 & $\mathrm{R}$ & 3 & Insectívoro \\
\hline \multicolumn{5}{|l|}{ Emberizidae } \\
\hline Atlapetes albinucha & 0.66 & $\mathrm{R}$ & 2.5 & Semillero \\
\hline Chlorospingus ophthalmicus & 0.08 & MA & 2 & Frugívoro pequeño \\
\hline Melozone cabanisi & 0.93 & $\mathrm{R}$ & 2.5 & Semillero \\
\hline Melozone leucotis & 5.21 & $\mathrm{R}$ & 2.5 & Semillero \\
\hline Zonotrichia capensis & 4.00 & $\mathrm{R}$ & 3 & Semillero \\
\hline \multicolumn{5}{|l|}{ Fringillidae } \\
\hline Chlorophonia callophrys & 0.47 & MA & 1.5 & Frugívoro pequeño \\
\hline Euphonia elegantisima & 0.02 & MA & 2 & Frugívoro pequeño \\
\hline Euphonia leuticapilla & 0.02 & $\mathrm{R}$ & 2.5 & Frugívoro pequeño \\
\hline \multicolumn{5}{|l|}{ Hirundinidae } \\
\hline Hirundo rustica & 0.93 & $\mathrm{MN}$ & 3 & Insectívoro \\
\hline Petrochelidon pyrrhonota & 0.35 & $\mathrm{MN}$ & 3 & Insectívoro \\
\hline Pygochelidon cyanoleuca & 15.03 & $\mathrm{R}$ & 3 & Insectívoro \\
\hline Riparia riparia & 0.28 & $\mathrm{MN}$ & 3 & Insectívoro \\
\hline Stelgidopteryx ruficollis & 0.12 & $\mathrm{R}$ & 3 & Insectívoro \\
\hline Stelgidopteryx serripennis & 0.09 & $\mathrm{R}$ & 3 & Insectívoro \\
\hline \multicolumn{5}{|l|}{ Icteridae } \\
\hline Dives dives & 2.65 & & 3 & Frugívoro pequeño \\
\hline Icterus galbula & 5.10 & $\mathrm{R}$ & 2.5 & Frugívoro pequeño \\
\hline Icterus spurius & 0.06 & $\mathrm{MN}$ & 3 & Frugívoro pequeño \\
\hline Molothrus aeneus & 0.67 & $\mathrm{R}$ & 3 & Frugívoro pequeño \\
\hline Psarocolius montezuma & 0.01 & $\mathrm{R}$ & 3 & Frugívoro grande \\
\hline Quiscalus mexicanus & 1.85 & $\mathrm{R}$ & 3 & Omnivoro \\
\hline Sturnella magna & 1.66 & $\mathrm{R}$ & 3 & Semillero \\
\hline \multicolumn{5}{|l|}{ Mimidae } \\
\hline Dumetella carolinensis & 0.01 & $\mathrm{MN}$ & 3 & Frugívoro pequeño \\
\hline \multicolumn{5}{|l|}{ Momotidae } \\
\hline Momotus lessonii & 2.56 & $\mathrm{R}$ & 2.5 & Omnívoro \\
\hline \multicolumn{5}{|l|}{ Odontophoridae } \\
\hline Colinus leucopogon & 2.89 & $\mathrm{R}$ & 2.5 & Semillero \\
\hline Dendrortyx leucoprys & 0.04 & $\mathrm{R}$ & 2 & Semillero \\
\hline \multicolumn{5}{|l|}{ Parulidae } \\
\hline Basileuterus rufifrons & 5.73 & $\mathrm{R}$ & 2 & Insectívoro \\
\hline Cardellina pusilla & 0.83 & $\mathrm{MN}$ & 2.5 & Insectívoro \\
\hline Geothlypis poliocephala & 0.03 & $\mathrm{R}$ & 3 & Insectívoro \\
\hline Icteria virens & 0.01 & $\mathrm{MN}$ & 3 & Insectívoro \\
\hline Mniotilta varia & 0.24 & $\mathrm{MN}$ & 2 & Insectívoro \\
\hline Oporornis formosus & 0.02 & $\mathrm{MN}$ & 1 & Insectívoro \\
\hline
\end{tabular}


CUADRO 1 (Continuación) / TABLE 1 (Continued)

\begin{tabular}{|c|c|c|c|c|}
\hline Taxa & Abundancia & *Residencia & Dependencia del Bosque & Tipo de Dieta \\
\hline Oporornis philadelphia & 0.10 & $\mathrm{MN}$ & 2.5 & Insectívoro \\
\hline Oreothlypis peregrina & 3.72 & $\mathrm{MN}$ & 2 & Insectívoro \\
\hline Parkesia motacilla & 0.01 & $\mathrm{MN}$ & 3 & Insectívoro \\
\hline Protonotaria citrea & 0.07 & $\mathrm{MN}$ & 2 & Insectívoro \\
\hline Seiurus aurocapilla & 0.04 & $\mathrm{MN}$ & 2 & Insectívoro \\
\hline Setophaga cerulea & 0.02 & $\mathrm{MN}$ & 2 & Insectívoro \\
\hline Setophaga discolor & 0.01 & $\mathrm{MN}$ & 2.5 & Insectívoro \\
\hline Setophaga fusca & 0.26 & $\mathrm{MN}$ & 2 & Insectívoro \\
\hline Setophaga pensylvanica & 0.34 & $\mathrm{MN}$ & 2.5 & Insectívoro \\
\hline Setophaga petechia & 0.31 & $\mathrm{MN}$ & 3 & Insectívoro \\
\hline Setophaga pinus & 0.01 & $\mathrm{MN}$ & 2.5 & Insectívoro \\
\hline Setophaga ruticilla & 0.04 & $\mathrm{MN}$ & 2.5 & Insectívoro \\
\hline Setophaga townsendi & 0.01 & $\mathrm{MN}$ & 2.5 & Insectívoro \\
\hline Setophaga virens & 0.01 & $\mathrm{MN}$ & 2 & Insectívoro \\
\hline Vermivora chrysoptera & 0.02 & $\mathrm{MN}$ & 2.5 & Insectívoro \\
\hline Vermivora chrysopteraXpinus & 0.03 & $\mathrm{MN}$ & 2.5 & Insectívoro \\
\hline Wilsonia canadensis & 0.12 & $\mathrm{MN}$ & 2.5 & Insectívoro \\
\hline \multicolumn{5}{|l|}{ Picidae } \\
\hline Colaptes rubiginosus & 0.01 & $\mathrm{R}$ & 2 & Frugívoro pequeño \\
\hline Dryocopus lineatus & 0.17 & $\mathrm{R}$ & 2.5 & Frugívoro grande \\
\hline Melanerpes hoffmannii & 8.12 & $\mathrm{R}$ & 2.5 & Frugívoro pequeño \\
\hline \multicolumn{5}{|l|}{ Pipridae } \\
\hline Chiroxiphia linearis & 0.56 & $\mathrm{R}$ & 2 & Frugívoro pequeño \\
\hline \multicolumn{5}{|l|}{ Psittacidae } \\
\hline Amazona albifrons & 0.06 & $\mathrm{R}$ & 2 & Frugívoro grande \\
\hline Brotogeris jugularis & 0.10 & $\mathrm{R}$ & 3 & Frugívoro pequeño \\
\hline Pionus senilis & 0.28 & $\mathrm{R}$ & 2 & Frugívoro grande \\
\hline Psittacara finschi & 18.46 & $\mathrm{R}$ & 3 & Frugívoro grande \\
\hline \multicolumn{5}{|l|}{ Rallidae } \\
\hline Aramides cajaneus & 0.01 & $\mathrm{R}$ & 2 & Omnívoro \\
\hline \multicolumn{5}{|l|}{ Striigidae } \\
\hline Glaucidium brasilianum & 0.29 & $\mathrm{R}$ & 2.5 & Cazador \\
\hline Megascops choliba & 0.03 & $\mathrm{R}$ & 3 & Cazador \\
\hline \multicolumn{5}{|l|}{ Thamnophilidae } \\
\hline Thamnophilus doliatus & 0.01 & $\mathrm{R}$ & 2.5 & Insectívoro \\
\hline \multicolumn{5}{|l|}{ Thraupidae } \\
\hline Cyanerpes cyaneus & 0.01 & $\mathrm{R}$ & 2 & Frugívoro pequeño \\
\hline Saltator atriceps & 0.01 & $\mathrm{R}$ & 3 & Frugívoro pequeño \\
\hline Saltator coerulescens & 2.54 & $\mathrm{R}$ & 3 & Frugívoro pequeño \\
\hline Saltator maximus & 0.24 & $\mathrm{R}$ & 3 & Frugívoro pequeño \\
\hline Tangara larvata & 0.02 & $\mathrm{R}$ & 3 & Frugívoro pequeño \\
\hline Thraupis episcopus & 12.88 & $\mathrm{R}$ & 3 & Frugívoro pequeño \\
\hline Thraupis palmarum & 0.01 & $\mathrm{R}$ & 3 & Frugívoro pequeño \\
\hline Tiaris olivacea & 0.10 & $\mathrm{R}$ & 3 & Semillero \\
\hline Volatinia jacarina & 0.03 & $\mathrm{R}$ & 3 & Semillero \\
\hline \multicolumn{5}{|l|}{ Tityridae } \\
\hline Pachyramphus aglaiae & 0.02 & $\mathrm{R}$ & 2.5 & Frugívoro pequeño \\
\hline Tityra semifasciata & 0.74 & $\mathrm{R}$ & 2.5 & Frugívoro pequeño \\
\hline
\end{tabular}


CUADRO 1 (Continuación) / TABLE 1 (Continued)

\begin{tabular}{|c|c|c|c|c|}
\hline Taxa & Abundancia & *Residencia & Dependencia del Bosque & Tipo de Dieta \\
\hline \multicolumn{5}{|l|}{ Trochilidae } \\
\hline Amazilia saucerrottei & 0.39 & $\mathrm{R}$ & 2.5 & Nectarívoro \\
\hline Amazilia tzacatl & 2.74 & $\mathrm{R}$ & 3 & Nectarívoro \\
\hline Campylopterus hemileucurus & 0.07 & MA & 2 & Nectarívoro \\
\hline Chlorostilbon canivetii & 0.10 & MA & 3 & Nectarívoro \\
\hline Eugenes fulgens & 0.02 & MA & 2 & Nectarívoro \\
\hline Heliodoxa jacula & 0.01 & MA & 2 & Nectarívoro \\
\hline Heliomaster constantii & 0.01 & MA & 2.5 & Nectarívoro \\
\hline \multicolumn{5}{|l|}{ Troglodytidae } \\
\hline Campylorhynchus rufinucha & 0.07 & $\mathrm{R}$ & 2.5 & Insectívoro \\
\hline Cantorchilus modestus & 3.16 & $\mathrm{R}$ & 3 & Insectívoro \\
\hline Henicorhina leucophrys & 0.03 & $\mathrm{R}$ & 1 & Insectívoro \\
\hline Thryophilus rufalbus & 0.16 & $\mathrm{R}$ & 1.5 & Insectívoro \\
\hline Troglodytes aedon & 5.58 & $\mathrm{R}$ & 3 & Insectívoro \\
\hline \multicolumn{5}{|l|}{ Turdidae } \\
\hline Catharus aurantiirostris & 1.90 & $\mathrm{R}$ & 2.5 & Frugívoro pequeño \\
\hline Catharus frantzii & 0.03 & MA & 2.5 & Frugívoro pequeño \\
\hline Catharus minimus & 0.01 & $\mathrm{MN}$ & 2.5 & Frugívoro pequeño \\
\hline Catharus ustulatus & 0.58 & $\mathrm{MN}$ & 2 & Frugívoro pequeño \\
\hline Hylocinchla mustelina & 0.33 & $\mathrm{MN}$ & 1.5 & Frugívoro pequeño \\
\hline Turdus grayi & 26.91 & $\mathrm{R}$ & 3 & Frugívoro pequeño \\
\hline Turdus plebejus & 0.17 & MA & 2 & Frugívoro pequeño \\
\hline \multicolumn{5}{|l|}{ Tyrannidae } \\
\hline Contopus cinereus & 0.11 & $\mathrm{MN}$ & 3 & Insectívoro \\
\hline Contopus cooperi & 0.20 & $\mathrm{MN}$ & 2.5 & Insectívoro \\
\hline Contopus sordidulus & 0.73 & $\mathrm{MN}$ & 2 & Insectívoro \\
\hline Contopus virens & 0.64 & $\mathrm{MN}$ & 2.5 & Insectívoro \\
\hline Elaenia flavogaster & 1.15 & $\mathrm{R}$ & 3 & Frugívoro pequeño \\
\hline Elaenia frantzii & 0.09 & MA & 2.5 & Frugívoro pequeño \\
\hline Empidonax flaviventris & 0.02 & $\mathrm{MN}$ & 2 & Insectívoro \\
\hline Empidonax minimus & 0.03 & $\mathrm{MN}$ & 2.5 & Insectívoro \\
\hline Empidonax traillii & 0.02 & $\mathrm{MN}$ & 2.5 & Insectívoro \\
\hline Empidonax alnorum & 0.06 & $\mathrm{MN}$ & 2.5 & Insectívoro \\
\hline Empidonax virecens & 0.15 & $\mathrm{MN}$ & 2 & Insectívoro \\
\hline Legatus leucophaius & 0.31 & MS & 3 & Insectívoro \\
\hline Megarhynchus pitangua & 3.36 & $\mathrm{R}$ & 2.5 & Frugívoro grande \\
\hline Mionectes oleagineus & 0.07 & $\mathrm{R}$ & 2 & Frugívoro pequeño \\
\hline Mionectes olivaceus & 0.16 & MA & 2 & Frugívoro pequeño \\
\hline Myiarchus crinitus & 0.11 & $\mathrm{MN}$ & 2 & Insectívoro \\
\hline Myiarchus tuberculifer & 0.02 & $\mathrm{R}$ & 2.5 & Insectívoro \\
\hline Myiarchus tyrannulus & 0.02 & $\mathrm{R}$ & 2.5 & Insectívoro \\
\hline Myiodynastes luteiventris & 0.29 & MS & 2.5 & Frugívoro pequeño \\
\hline Myiodynastes maculatus & 0.01 & MS & 2.5 & Frugívoro pequeño \\
\hline Myiozetetes similis & 1.47 & $\mathrm{R}$ & 3 & Frugívoro pequeño \\
\hline Pitangus sulphuratus & 3.72 & $\mathrm{R}$ & 3 & Frugívoro pequeño \\
\hline Todirostrum cinereum & 0.01 & $\mathrm{R}$ & 3 & Frugívoro pequeño \\
\hline Tyrannus melancholicus & 6.40 & $\mathrm{R}$ & 3 & Frugívoro pequeño \\
\hline Tyrannus tyrannus & 0.18 & $\mathrm{MN}$ & 3 & Frugívoro pequeño \\
\hline
\end{tabular}


CUADRO 1 (Continuación) / TABLE 1 (Continued)

\begin{tabular}{lcccc}
\multicolumn{1}{c}{ Taxa } & Abundancia & $*$ Residencia & Dependencia del Bosque & Tipo de Dieta \\
$\begin{array}{l}\text { Tyrannus verticalis } \\
\text { Vireonidae }\end{array}$ & 0.20 & $\mathrm{MN}$ & 3 & Frugívoro pequeño \\
Vireo flavifrons & 0.33 & $\mathrm{MN}$ & 2 & Frugívoro pequeño \\
Vireo flavoviridis & 1.27 & $\mathrm{MS}$ & 3 & Frugívoro pequeño \\
Vireo gilvus & 0.01 & $\mathrm{MN}$ & 2.5 & Frugívoro pequeño \\
Vireo leucophrys & 0.13 & $\mathrm{R}$ & 3 & Frugívoro pequeño \\
Vireo olivaceus & 1.71 & $\mathrm{MN}$ & 3 & Frugívoro pequeño \\
Vireo philadelphicus & 0.19 & $\mathrm{MN}$ & 2.5 & Frugívoro pequeño \\
\hline
\end{tabular}

La abundancia corresponde a la cantidad de individuos promedios detectados en 89 muestreos. Las especies están clasificadas según su tipo de residencia en el país, dependencia del bosque, y tipo de dieta. *Residencia: $\mathrm{R}=\mathrm{Residente,}$ $\mathrm{MN}=$ Migratoria de Norte América, MA = Especie residente con migración altitudinal, MS = Migratoria de Sur América. Abundance is the mean number of individuals per sampling day for a total of 89 samples. Each species was classified according to resident status in the country, forest dependence, and food guild. *Resident status: $\mathrm{R}=$ Resident, $\mathrm{MN}=\mathrm{North}$ America Migratory, MA = Resident species with altitudinal migration, MS = South America Migratory.

cuatro sureñas) y 101 residentes (de las cuales 13 fueron migratorias altitudinales). La riqueza total de especies (MLM: $\mathrm{F}_{6,68}=2.93, \mathrm{P}=$ $0.01)$ y de especies residentes $\left(\mathrm{F}_{6,68}=3.93, \mathrm{P}\right.$ $=0.002$ ) varió entre años y en ambos casos fue menor durante 2004 (Cuadro 2). Sin embargo, la riqueza de especies migratorias altitudinales $\left(\mathrm{F}_{5,20}=1.46, \mathrm{P}=0.25\right)$, migratorias norteñas $\left(\mathrm{F}_{7,63}=1.86, \mathrm{P}=0.09\right)$, y migratorias sureñas $\left(\mathrm{F}_{7,20}=1.73, \mathrm{P}=0.16\right)$ fue similar entre todos años (Cuadro 2).

La riqueza total de especies fue mayor en enero, febrero, marzo, y setiembre; pero menor en junio y diciembre $\left(\mathrm{F}_{11,68}=2.85, \mathrm{P}=0.004\right.$;
Cuadro 3). La riqueza de especies residentes fue mayor en enero, febrero, y marzo; pero menor en diciembre $\left(\mathrm{F}_{11,67}=2.32, \mathrm{P}=0.02\right.$; Cuadro 3). La riqueza de especies migratorias altitudinales fue mayor en enero y febrero; pero menor en octubre y noviembre cuando no detecté ninguna de estas especies $\left(\mathrm{F}_{9,19}=2.96\right.$, $\mathrm{P}=0.02$; Cuadro 3). Aunque detecté especies migratorias norteñas durante todo el año, la riqueza de estas especies fue mayor de setiembre a abril, y menor el resto del año $\left(\mathrm{F}_{11,58}=\right.$ $4.68, \mathrm{P}<0.001$; Cuadro 3). La riqueza de especies migratorias sureñas fue similar entre todos los meses $\left(\mathrm{F}_{7,29}=0.39, \mathrm{P}=0.90 ;\right.$ Cuadro 3$)$.

CUADRO 2

Número de especies de aves promedio ( \pm error estándar) por año según el tipo de residencia en el país, dentro de una matriz agrícola rural en Getsemaní, Heredia, Costa Rica

TABLE 2

Bird species mean ( \pm standard error) per year according to resident status in the country, inside 30 ha of agricultural rural matrix at Getsemaní, Heredia, Costa Rica

\begin{tabular}{cccccc} 
Año & Todos & Residentes & Migratorio Altitudinal & Migratorio Norteño & Migratorio Sureños \\
2003 & $36.41 \pm 1.84$ & $30.61 \pm 1.18$ & $1.58 \pm 0.32$ & $5.78 \pm 1.46$ & $1.37 \pm 0.19$ \\
2004 & $32.84 \pm 1.90$ & $27.73 \pm 1.23$ & $1.16 \pm 0.42$ & $6.07 \pm 1.05$ & $1.04 \pm 0.27$ \\
2005 & $38.89 \pm 3.50$ & $30.65 \pm 2.46$ & $0 \pm 0$ & $2.62 \pm 1.38$ & $1.01 \pm 0.3$ \\
2006 & $39.87 \pm 1.99$ & $33.48 \pm 1.33$ & $2.17 \pm 0.37$ & $6.49 \pm 1.31$ & $1.68 \pm 0.25$ \\
2007 & $41.68 \pm 2.80$ & $35.26 \pm 1.97$ & $1.58 \pm 0.46$ & $6.41 \pm 1.25$ & $1.01 \pm 0.39$ \\
2008 & $43.00 \pm 4.08$ & $37.03 \pm 2.97$ & $1.22 \pm 0.49$ & $7.22 \pm 1.96$ & $1.31 \pm 0.4$ \\
2009 & $41.01 \pm 3.45$ & $35.56 \pm 2.41$ & $1.77 \pm 0.56$ & $5.82 \pm 2.01$ & $1.78 \pm 0.32$ \\
2010 & & & & $4.61 \pm 1.42$ & \\
\hline
\end{tabular}


CUADRO 3

Número de especies de aves promedio ( \pm error estándar) por mes (2002 a 2010) según el tipo de residencia en el país, dentro de una matriz agrícola rural, Getsemaní, Heredia, Costa Rica

TABLE 3

Bird species mean ( \pm standard error) per month (2002 to 2010) according to resident status in the country, inside of agricultural rural matrix at Getsemaní, Heredia, Costa Rica

\begin{tabular}{lccccc}
\multicolumn{1}{c}{ Mes } & Todos & Residentes & Migratorio Altitudinal & Migratorio Norteño & Migratorio Sureño \\
Enero & $42.60 \pm 4.53$ & $36.00 \pm 3.18$ & $1.60 \pm 0.98$ & $6.60 \pm 1.63$ & \\
Febrero & $44.75 \pm 1.11$ & $35.75 \pm 1.6$ & $1.75 \pm 0.48$ & $9.00 \pm 0.82$ & $0.73 \pm 0.27$ \\
Marzo & $42.36 \pm 1.52$ & $34.36 \pm 1.14$ & $1.00 \pm 0.36$ & $7.27 \pm 0.97$ & $0.70 \pm 0.30$ \\
Abril & $36.30 \pm 2.08$ & $28.30 \pm 1.27$ & $0.70 \pm 0.21$ & $7.30 \pm 1.09$ & $0.86 \pm 0.34$ \\
Mayo & $33.57 \pm 2.19$ & $30.86 \pm 1.98$ & $0.43 \pm 0.30$ & $0.71 \pm 0.52$ & $1.00 \pm 0.41$ \\
Junio & $29.00 \pm 2.68$ & $27.75 \pm 2.50$ & $0.75 \pm 0.48$ & $0.33 \pm 0.21$ & $1.50 \pm 0.43$ \\
Julio & $32.83 \pm 0.91$ & $30.83 \pm 0.91$ & $0.33 \pm 0.21$ & $1.50 \pm 0.43$ & $1.10 \pm 0.28$ \\
Agosto & $36.10 \pm 1.68$ & $33.50 \pm 1.49$ & $0.70 \pm 0.26$ & $7.07 \pm 1.03$ & $0.67 \pm 0.21$ \\
Setiembre & $39.93 \pm 2.29$ & $32.27 \pm 1.86$ & $0.33 \pm 0.13$ & $6.57 \pm 1.04$ & $0.43 \pm 0.20$ \\
Octubre & $34.43 \pm 1.97$ & $27.43 \pm 1.15$ & $0 \pm 0$ & $7.75 \pm 2.43$ & $6.50 \pm 1.59$ \\
Noviembre & $35.25 \pm 4.42$ & $27.50 \pm 2.40$ & $0 \pm 0$ & $0.33 \pm 0.33$ & \\
Diciembre & $29.67 \pm 3.67$ & $22.50 \pm 3.01$ & &
\end{tabular}

Encontré que la riqueza y abundancia de especies fue similar entre los meses al comparar todas las especies (ANOSIM: $\mathrm{R}=0.07, \mathrm{P}=$ 0.16 ; Fig. 2), las especies residentes $(\mathrm{R}=0.02$, $\mathrm{P}=0.34$; Fig. 2), y las migratorias altitudinales $(\mathrm{R}=0.10, \mathrm{P}=0.14$; Fig. 2$)$. Pero existió variación de la riqueza y abundancia de las especies migratorias, tanto norteñas como sureñas, por mes. Los muestreos en setiembre presentaron una mayor similitud de riqueza y abundancia de especies migratorias norteñas entre sí que con los otros meses $(\mathrm{R}=0.40, \mathrm{P}<0.001$; Fig. $2)$; mientras que la riqueza y abundancia de especies migratorias sureñas fue más similar dentro del mes de julio y agosto, que entre los otros meses ( $\mathrm{R}=0.21, \mathrm{P}=0.03$; Fig. 2 ).

La riqueza de especies por grado de dependencia al bosque, varió entre meses únicamente para aquellas especies que viven en áreas con al menos $50 \%$ de cobertura boscosa y clasificadas en las categoría 2 (ALG: $\mathrm{X}^{2}=41.52$, g.l. $=11, \mathrm{P}<0.001) \mathrm{y}$ aquellas especies que viven tanto en áreas con al menos $50 \%$ de cobertura boscosa y áreas abiertas que fueron clasificadas en la categoría $2.5\left(\mathrm{X}^{2}=25.29\right.$, g.l. $=11, \mathrm{P}=$ 0.008). Las especies de categoría 2 fueron más abundantes de enero a marzo y menos en junio y julio (Cuadro 4); mientras que las especies de categoría 2.5 fueron más abundantes en febrero y menos en junio (Cuadro 4). Para las especies más dependientes de bosque (categorías 1 y 1.5) no hubo diferencias entre la riqueza de especies por mes $\left(\mathrm{X}^{2}=5.32\right.$, g.l. $=11, \mathrm{P}=0.81$; Cuadro 4). Lo mismo concluí para las especies de zonas abiertas, donde la riqueza de especies fue igual entre meses $\left(X^{2}=11.72\right.$, g.1. $=11$, $\mathrm{P}=0.39$; Cuadro 4). Durante los ocho años de estudio únicamente registré dos aves asociadas a humedales (categoría 4): la Cigueña Americana Mycteria americana y el Chorlito Dorado Chico Pluvialis dominica (Cuadro 1), por lo que ningún análisis se realizo con estas especies.

$\mathrm{Al}$ analizar la riqueza de especies por tipo de dieta solo las frugívoras pequeñas (ALG: $\mathrm{X}^{2}$ $=23.86$, g.l. $=11, \mathrm{P}=0.01)$ e insectívoras $\left(\mathrm{X}^{2}\right.$ $=16.49$, g.l. $=11, \mathrm{P}<0.001)$ variaron según el mes. Detecté mayor cantidad de especies frugívoras pequeñas en enero y febrero (Cuadro 5); mientras que setiembre presentó la mayor riqueza de aves insectívoras (Cuadro 5). Para las demás categorías de aves según su dieta 

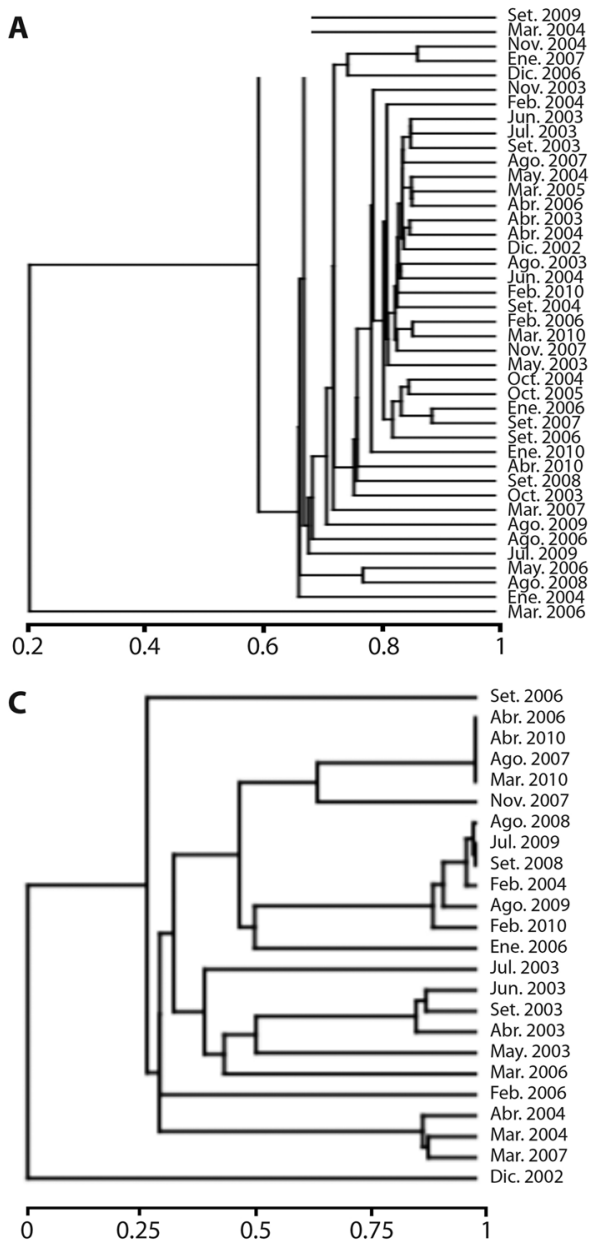

E

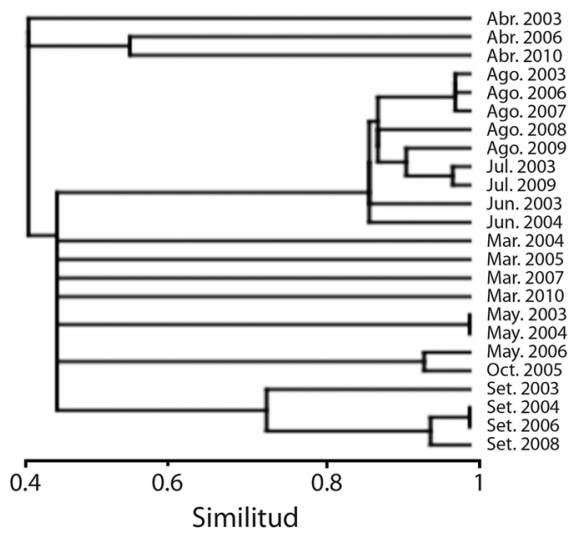

B
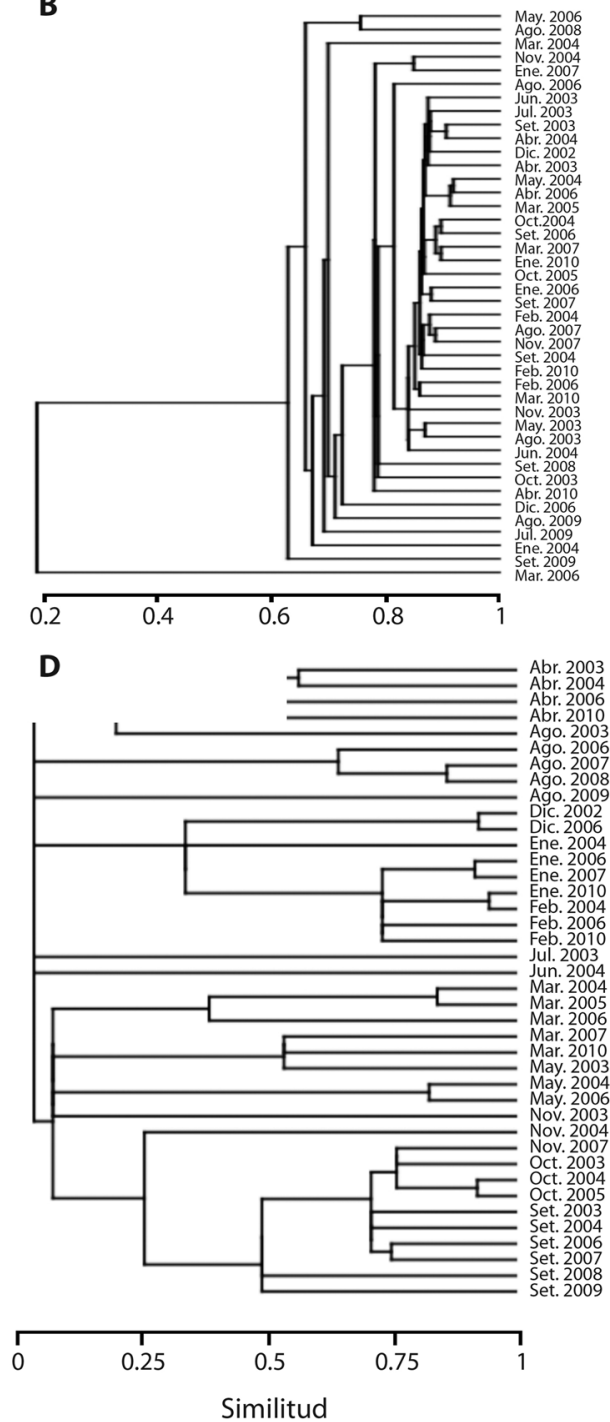

Fig. 2. Análisis de conglomerados tomando en cuenta la riqueza de especies de aves y su abundancia por mes de 2002 a 2010 , utilizando la distancia del índice de Morisita y el método de unión simple. Cada conglomerado representa un tipo de residencia en el país, y en que fueron clasificadas las aves: A) Todas las especies, B) Residentes, C) Migratorias Altitudinales, D) Migratorias Norteñas, y E) Migratorias Sureñas. Valores cercanos a 1 representan una mayor similitud entre la riqueza de especies y su abundancia.

Fig. 2. Cluster analysis including species richness and abundance by month from 2002 to 2010, using Morisita index of similarity with single linkage union method. Each shows the bird species status in the country: A) All species, B) Residents, C) Altitudinal Migrants, D) Northern Migrants, and E) Southern Migrants. Values close to 1 are high level of similarity in richness and abundance. 


\section{CUADRO 4}

Número de especies de aves promedio ( \pm error estándar) por mes (2002 a 2010) según el grado de dependencia de bosque dentro de una matriz agrícola rural en Getsemaní, Heredia, Costa Rica

TABLE 4

Bird species mean ( \pm standar error) per month (2002 to 2010) according to forest dependence inside of agricultural rural matrix sampled at Getsemaní, Heredia, Costa Rica

\begin{tabular}{lcccc}
\multicolumn{1}{r}{ Mes } & $1-1.5$ & 2 & 2.5 & 3 \\
Enero & $2.67 \pm 0.88$ & $6.80 \pm 1.36$ & $12.8 \pm 0.92$ & $21.40 \pm 1.81$ \\
Febrero & $1.25 \pm 0.25$ & $5.50 \pm 0.96$ & $14.75 \pm 1.25$ & $23.00 \pm 1.47$ \\
Marzo & $1.14 \pm 0.14$ & $5.27 \pm 0.54$ & $12.91 \pm 0.71$ & $23.45 \pm 0.64$ \\
Abril & $2.00 \pm 0.58$ & $4.50 \pm 0.58$ & $11.90 \pm 0.90$ & $19.20 \pm 1.31$ \\
Mayo & $1.00 \pm 0$ & $3.00 \pm 0.58$ & $10.00 \pm 0.93$ & $20.86 \pm 0.99$ \\
Junio & $0 \pm 0$ & $1.50 \pm 0.50$ & $8.25 \pm 1.31$ & $19.25 \pm 1.03$ \\
Julio & $1.00 \pm 0$ & $1.50 \pm 0.22$ & $9.00 \pm 0.68$ & $21.83 \pm 0.79$ \\
Agosto & $1.20 \pm 0.20$ & $2.89 \pm 0.26$ & $10.60 \pm 0.72$ & $22.30 \pm 1.01$ \\
Setiembre & $1.00 \pm 0$ & $3.93 \pm 0.44$ & $12.93 \pm 0.86$ & $22.80 \pm 1.23$ \\
Octubre & $1.00 \pm 0$ & $3.57 \pm 0.81$ & $9.14 \pm 0.70$ & $21.57 \pm 0.92$ \\
Noviembre & $0 \pm 0$ & $4.25 \pm 1.03$ & $10.75 \pm 1.89$ & $20.25 \pm 1.70$ \\
Diciembre & $2.00 \pm 0$ & $3.00 \pm 0.71$ & $9.00 \pm 0.82$ & $17.83 \pm 2.36$ \\
\hline
\end{tabular}

Valores de 1 y 1.5 son muy dependientes de bosque y valores de 3 nada dependiente de bosque.

Values of 1 and 1.5 are species highly dependent of forest, and values of 3 are species no-dependent of forest.

\section{CUADRO 5}

Número de especies de aves promedio ( \pm error estándar) por mes (2002 a 2010) según el tipo de dieta, de una matriz agrícola rural en Getsemaní, Heredia, Costa Rica

TABLE 5

Bird species mean ( \pm standar error) per month (2002 to 2010) according to food guild, inside of agricultural rural matrix at Getsemaní, Heredia, Costa Rica

\begin{tabular}{lccccccc}
\multicolumn{1}{c}{ Mes } & Cazadores & Frugívoros Grandes & Frugívoros Pequeños & Insectívoros & Nectarívoros & Omnívoro & Semilleros \\
Enero & $3.20 \pm 0.73$ & $3.20 \pm 0.58$ & $15.40 \pm 2.29$ & $9.80 \pm 1.36$ & $2.00 \pm 0$ & $2.00 \pm 0.45$ & $7.00 \pm 0.89$ \\
Febrero & $3.00 \pm 0.41$ & $3.00 \pm 0$ & $15.75 \pm 0.85$ & $11.75 \pm 0.95$ & $1.75 \pm 0.48$ & $2.25 \pm 0.48$ & $7.25 \pm 1.03$ \\
Marzo & $3.18 \pm 0.40$ & $3.18 \pm 0.18$ & $13.27 \pm 0.89$ & $10.27 \pm 0.89$ & $1.64 \pm 0.15$ & $2.45 \pm 0.16$ & $8.36 \pm 0.51$ \\
Abril & $1.78 \pm 0.22$ & $2.56 \pm 0.18$ & $11.80 \pm 1.09$ & $11.20 \pm 0.84$ & $1.63 \pm 0.18$ & $1.70 \pm 0.21$ & $6.40 \pm 0.52$ \\
Mayo & $2.67 \pm 0.33$ & $3.14 \pm 0.34$ & $9.86 \pm 0.74$ & $8.14 \pm 0.77$ & $1.86 \pm 0.34$ & $1.17 \pm 0.17$ & $7.29 \pm 0.81$ \\
Junio & $1.75 \pm 0.48$ & $2.00 \pm 0.41$ & $9.50 \pm 1.32$ & $7.00 \pm 0.71$ & $1.67 \pm 0.67$ & $1.00 \pm 0$ & $6.75 \pm 0.25$ \\
Julio & $1.80 \pm 0.20$ & $3.17 \pm 0.31$ & $9.83 \pm 0.31$ & $8.17 \pm 0.79$ & $1.20 \pm 0.20$ & $1.40 \pm 0.24$ & $8.00 \pm 0.68$ \\
Agosto & $2.30 \pm 0.40$ & $3.00 \pm 0.33$ & $11.00 \pm 0.63$ & $9.30 \pm 0.72$ & $1.30 \pm 0.21$ & $1.44 \pm 0.18$ & $7.90 \pm 0.66$ \\
Setiembre & $3.00 \pm 0.32$ & $2.80 \pm 0.24$ & $10.53 \pm 0.79$ & $14.00 \pm 1.07$ & $1.50 \pm 0.15$ & $1.53 \pm 0.13$ & $7.27 \pm 0.38$ \\
Octubre & $3.00 \pm 0.45$ & $2.71 \pm 0.36$ & $10.43 \pm 1.02$ & $10.57 \pm 0.78$ & $1.00 \pm 0$ & $1.57 \pm 0.20$ & $5.86 \pm 0.63$ \\
Noviembre & $2.50 \pm 0.29$ & $2.50 \pm 0.29$ & $10.50 \pm 2.63$ & $11.50 \pm 1.19$ & $1.67 \pm 0.67$ & $1.25 \pm 0.25$ & $5.75 \pm 0.25$ \\
Diciembre & $1.83 \pm 0.48$ & $2.50 \pm 0.34$ & $9.50 \pm 0.89$ & $9.00 \pm 1.81$ & $1.50 \pm 0.34$ & $1.40 \pm 0.24$ & $4.17 \pm 0.83$ \\
\hline
\end{tabular}


(cazadores, frugívoros grandes, nectarívoros, omnívoros, y semilleros) la riqueza fue similar entre meses $(\mathrm{P}>0.14$ para todos, Cuadro 5).

\section{DISCUSIÓN}

La riqueza de aves registrada en Getsemaní durante ocho años de estudio fue alta en comparación a otros sitios agrícolas y urbanos relativamente cercanos (ej.: Los Chigüites, Parque La Libertad, Centro Conservación Santa Ana, Universidad de Costa Rica) (Naoki, Duran, \& Sánchez, 2003; Alvarado-Quesada \& Bolaños-Redondo, 2010; Solano-Ugalde, 2011; Biamonte et al., 2011). Además, la riqueza de especies registradas en el área representa un $17.5 \%$ de toda la riqueza de aves del país (N = 920; Sandoval \& Sánchez, 2017), un $19.7 \%$ de las aves terrestres del país, y un $34.9 \%$ de las aves migratorias latitudinales (Sandoval \& Sánchez, 2017).

Los datos de riqueza demuestran que las matrices agrícolas que mezclan cafetales con sombra y sol, charrales o tacotales (estadios tempranos de sucesión vegetal), potreros o pasturas, bosques de galería, y zonas urbanorurales son importantes para la ocurrencia de muchas especies de aves residentes y migratorias. Mis resultados concuerdan con lo reportado en sistemas de café con sombra (Wunderle \& Latta, 1996; Johnson, 2000) y plantaciones de cacao (Reitsma, Parrish, \& McLarney, 2001; Faria, Laps, Baumgarten, \& Cetra, 2006). La riqueza de aves en las matrices mixtas son mayores a la riqueza de las matrices homogéneas, como por ejemplo pastizales, plantaciones de palma aceitera, y piñales, tanto para aves residentes como migratorias (López-Ricuarte, Edwards, Romero-Rodríguez, \& Gilroy, 2017).

Los pocos cambios que sufrió la estructura del hábitat del sitio de estudio durante los ocho años de muestreo, pueden explicar porqué la riqueza de especies entre años fue similar (en 7 de 8 años), y no hubo un patrón de aumento o disminución de especies entre años. Posiblemente para detectar cambios de especies dentro de estos ambientes agrícolas-rurales con pocos cambios en la cobertura vegetal es necesario mayores periodos de tiempo, mayores cambios en la matriz circundante, o aumento de la cobertura boscosa como ha ocurrido en otros sitios agrícolas que sí reportan cambios en la riqueza de especies a través del tiempo (Chamberlain, Fuller, Bunce, Duckworth, \& Shrubb, 2000; Magurran et al. 2010; Biamonte et al. 2011).

Fenológicamente, cada grupo de aves (según tipo de residencia, dependencia del bosque y dieta) presentó un comportamiento diferente a lo largo del año. Sin embargo, para la mayoría de las categorías de especies donde varió la riqueza por meses, la mayor riqueza ocurrió de enero a marzo, que corresponde con la época seca en el área de estudio (Sandoval, 2011). La mayor riqueza de especies de enero a marzo por tipo de residencia puede ser el resultado de la mezcla de las aves residentes, con las migratorias norteñas, y los residentes que realizan migración altitudinal durante esos meses como lo registré.

Por su parte, de diciembre a marzo es común que en las partes altas de las montañas en Costa Rica y en especial las que dividen la vertiente Caribe de la Pacífico presenten condiciones climáticas adversas para las aves, como fuertes vientos, lluvias constantes y bajas temperaturas (obs. pers.). Esto favorece que varias especies de aves (ej.: Paloma Collareja Patagioenas fasciata, Pitorreal Ptiliogonys caudatus, o Rualdo Chlorophonia callophrys) se desplacen de las zonas altas a las zonas bajas aledañas en busca de mejores condiciones climáticas o alimento (Avalos, 2005), por lo que aumenta la probabilidad de encontrar una mayor riqueza de especies a menor altitud. Por otro lado, aunque de enero a marzo ya no hay aves migratorias desplazándose de norte a sur, que es el momento de mayor riqueza de este grupo de especies en el país (Stiles \& Skutch, 1989; Stiles, 1994); la riqueza de aves migratorias en el área de estudio fue constante durante los ocho meses que hibernan en el país (Cuadro 2), contribuyendo durante ese periodo a la riqueza total del sitio de estudio.

Un aumento en la riqueza de especies con dependencia intermedia del bosque (categorías 
2 y 2.5) de enero a marzo se puede atribuir a que la mayoría de aves migratorias altitudinales y norteñas que se encontraron en el área de estudio son clasificadas en estas categorías (Stiles, 1985; Biamonte et al., 2011), y para estos meses es cuando hay una mayor riqueza de especies en el área. Mientras que las especies con grado de dependencia alta (categorías 1 y 1.5) y baja del bosque (categoría 3) no presentaron cambios en la abundancia de riqueza entre meses. Las especies con alto grado de dependencia del bosque son raras en el área de estudio, debido a que las áreas grandes de bosque son escasas en los alrededores. La falta de bosques maduros primarios o secundarios son el resultado principalmente de una gran expansión cafetalera y agrícola al interior del Valle Central de Costa Rica y posteriormente al desarrollo urbanístico que disminuyó el área de los bosques naturales y las conexiones entre los bosques remanentes (Stiles, 1990; Biamonte et al., 2011). Finalmente, que la riqueza de aves poco dependientes de bosque (categoría 3) fuera constante entre los meses, se explica porque el recurso que utilizan estas especies fue constante todo el año.

Al analizar la riqueza de especies por el tipo de dieta los frugívoros pequeños variaron su riqueza a lo largo del año, aumentando de enero a marzo. Lo anterior se puede deber a que, durante el periodo seco en el área de estudio es común encontrar árboles como higuerones Ficus sp. (Moraceae), arbustos y epífitas (ej.: Loranthaceae) en su pico de fructificación (obs. pers.), favoreciendo la ocurrencia de especies en esta categoría como Rualdo, Veraneros (Euphonia elegantisima), Yigüirros de Montaña (Turdus plebejus), Siete Colores (Tangara larvata), Cardenales (Piranga rubra), o Caciques (Icterus galbula) que pueden aprovechar la abundancia de esos frutos (Stiles \& Skutch 1989). Pese a esta variación en frugívoros pequeños y su posible asociación con la abundancia de frutos, los frugívoros grandes no variaron su abundancia a lo largo del año. Esto se podría deber a que en general estas especies son menos comunes tanto dentro (como lo encontré) como alrededor del área de estudio, y por lo tanto pese a que su abundancia puede variar en respuesta a la presencia de frutos, estas variaciones son pequeñas y por lo tanto no fueron detectables en este estudio. Por otro lado que el gremio insectívoro fuera más abundante en setiembre esto porque la mayoría de aves migratorias norteñas son principalmente insectívoras y para este mes es cuando incrementa este grupo de especies en el área de estudio. La riqueza de aves en las otras categorías de dieta fue similar a lo largo del año, lo que puede indicar que el recurso alimenticio es constante durante el año en el sitio.

En conclusión, este estudio reveló diferentes patrones de riqueza de aves en un paisaje agrícola-rural a lo largo del año según el grado de dependencia del bosque, tipo de dieta, y residencia en el país. También demostró que las matrices agrícolas-rurales permiten la ocurrencia de una gran riqueza de especies residentes y migratorias. Lo anterior demuestra que estas matrices poseen una riqueza de especies muy alta y en constante variación a lo largo del año. Por lo tanto si se pretende generar políticas de conservación para mantener estos ambientes (ej.: evitar que se transformen en sitios urbanos) y las aves que los habitan se deben tomar en cuenta los cambios en los patrones de riqueza por grupo de especies ya que no todas varían de la misma forma.

Declaración de ética: el autor declara que está de acuerdo con esta publicación; que no existe conflicto de interés de ningún tipo; y que ha cumplido con todos los requisitos y procedimientos éticos y legales pertinentes. Todas las fuentes de financiamiento se detallan plena y claramente en la sección de agradecimientos. El respectivo documento legal firmado se encuentra en los archivos de la revista.

\section{AGRADECIMIENTOS}

A Olman Sandoval por acompañarme en muchos de los muestreos de este proyecto. A Diego Ocampo y a la increíble suma de seis revisores anónimos por lo comentarios previos a un borrador de este documento, y a Gilbert 
Barrantes por la discusión de los análisis estadísticos. El análisis y escritura de esta investigación fue realizada con un nombramiento de medio tiempo por parte de la Escuela de Biología. Finalmente quiero agradecer a Nelson Chaves y Álvaro Picado por toda la colaboración para pasar los datos de las libretas a la base de datos.

\section{RESUMEN}

Los mosaicos agrícolas han sido estudiados para comprender como contribuyen a mantener la riqueza de las especies según el tipo de matriz. Sin embargo, el estudio de la fenología de las especies dentro de estos ambientes ha sido dejado de lado. Por lo tanto mi objetivo con esta investigación fue analizar los cambios en la riqueza y abundancia de las especies de aves entre meses dentro de un mosaico periurbano agrícola tropical. Adicionalmente analicé si los cambios en la riqueza de las especies varían según su grado de dependencia por el bosque y el gremio alimenticio al que pertenecen. Realice muestreos mensuales del 2002 al 2010 dentro de una matriz de 30 ha dominada por potreros y cafetales, mezcladas con regeneración natural y zonas urbano-rurales. A lo largo de los muestreos anoté todos los individuos visto y escuchados de cada especie a lo largo de un trasecto de $3 \mathrm{~km}$. Encontré que la riqueza de especies varió a lo largo de los meses, siendo mayor de enero a marzo (periodo seco del área de estudio) para todas las categorías de residencia (ej.: residentes, migratorias norteñas, sureñas, y altitudinales). Por tipo de gremio alimenticio la riqueza de especies aumento de enero a febrero para los frugívoros pequeños y en setiembre para los insectívoros; mientras que los otros gremios presentaron una riqueza similar de especies a lo largo del año. Por grado de dependencia de bosque solo las aves con dependencia moderada presentaron un aumento en su riqueza de enero a junio. El estudio reveló la ocurrencia de diferentes patrones de riqueza de aves a lo largo del año según el grado de dependencia del bosque, gremio alimenticio, y residencia en el país. Estas matrices poseen una riqueza alta de especies, pero en constante variación a lo largo del año, por lo que estos cambios en los patrones de riqueza deberían tomarse en cuenta para futuras políticas de conservación de dichos agroecosistemas.

Palabras clave: abundancia de aves; cafetales; Costa Rica; uso de suelo; fenología de especies de aves; potreros; riqueza de aves.

\section{REFERENCIAS}

Alvarado-Quesada, G. M., \& Bolaños-Redondo, S. E. (2010). Importancia del Parque La Libertad para especies de aves poco comunes del Valle Central, Costa Rica. Brenesia, 73, 163-164.

Avalos, G. (2005). Banded-Tailed Pigeon (Columba fasciata) at low elevations in Braulio Carrillo National Park, Costa Rica. Ornitología Neotropical, 16, 127-128.

Barrantes, G., Ocampo, D., Ramírez-Fernández, J. D., \& Fuchs, E. J. (2016). Effect of fragmentation on the Costa Rican dry forest avifauna. PeerJ, 4, e2422.

Beninde, J., Feldmeier, S., Werner, M., Peroverde, D., Schulte, U., Hochkirch, A., \& Veith, M. (2016). Cityscape genetics: structural vs. functional connectivity of an urban lizard population. Molecular Ecology, 25, 4984-5000.

Biamonte, E., Sandoval, L., Chacón, E., \& Barrantes, G. (2011). Effect of urbanization on the avifauna in a tropical metropolitan area. Landscape Ecology, 26, 183-194.

Bierregaard, R. O., \& Lovejoy, T. E. (1988). Birds in Amazonian forest fragments: effects of insularization. Proceedings of International Ornithology Congress, 19, 1564-1579.

Chamberlain, D. E., Fuller, R. J., Bunce, R. G. H., Duckworth, J. C., \& Shrubb, M. (2000). Changes in the abundance of farmland birds in relation to the timing of agricultural intensification in England and Wales. Journal of Applied Ecology, 37, 771-788.

Costantini, D., Greives, T. J., Hau, M., \& Partecke, J. (2014). Does urban life change blood oxidative status in birds?, Journal of Experimental Biology, 217, 2994-2997.

Faria, D., Laps, R. R., Baumgarten, J., \& Cetra, M. (2006). Bat and bird assemblages from forests and shade cacao plantations in two contrasting landscapes in the Atlantic Forest of southern Bahia, Brazil. Biodiversity \& Conservation, 15, 587-612.

Fischer, J., Brosi, B., Daily, G. C., Ehrlich, P. R., Goldman, R., Goldstein, ..., Tallis, H. (2008). Should agricultural policies encourage land sparing or wildlife-friendly farming?. Frontiers in Ecology and the Environment, 6, 380-385.

Foley, J. A., DeFries, R., Asner, G.P., Barford, C., Bonan, G., Carpenter, ..., Snyder, P. K. (2005). Global consequences of land use. Science, 309, 570-574. 
Foley, J. A., Ramankutty, N., Brauman, K. A., Cassidy, E. S., Gerber, J. S., Johnston, ..., Zaks, D.P.M. (2011). Solutions for a cultivated planet. Nature, 478, 337-342.

Greenberg, R., Bichier, P., \& Sterling, J. (1997). Bird populations in rustic and planted shade coffee plantations of Eastern Chiapas, Mexico. Biotropica, 29, 501-514.

Greenberg, R., Bichier, P., Cruz Agnon, A., \& Reitsma, R. (1997). Bird Populations in shade and sun coffee plantations in Central Guatemala. Conservation Bio$\log y, 11,448-459$.

Hammer, Ø., Harper, D. A. T., \& Ryan, P. D. (2001) Past: Paleontological Statistics Software Package for Education and Data Analysis. Palaeontologia Electronica, 4(1), 1-9.

Hughes, J., Daily, G. C., \& Ehrlich, P. R. (1997). Population diversity: its extent and extinction. Science, 278, 689-692.

Johnson, M. D. (2000). Effects of shade-tree species and crop structure on the winter arthropod and bird communities in a Jamaican shade coffee plantation. Biotropica, 32, 133-145.

López-Ricaurte, L., Edwards, D. P., Romero-Rodríguez, N., \& Gilroy, J. J. (2017). Impacts of oil palm expansion on avian biodiversity in a Neotropical natural savanna. Biological Conservation, 213, 225-233.

Magurran, A. E., Baillie, S. R., Buckland, S. T., Dick, J. M., Elston, D. A., Scott, ..., Watt, A. D. (2010). Longterm datasets in biodiversity research and monitoring: assessing change in ecological communities through time. Trends in Ecology \& Evolution, 25, 574-582.

Manhães, M. A., \& Loures-Ribeiro, A. (2005). Spatial distribution and diversity of bird community in an urban area of southeast Brazil. Brazilian. Archives of Biology and Technology, 48, 285-294.

Meillère, A., Brischoux, F., Parenteau, C., \& Angelier, F. (2015). Influence of urbanization on body size, condition, and physiology in an urban exploiter: a multicomponent approach. PloS one, 10(8), e0135685.

Miles, L., \& Kapos, V. (2008). Reducing greenhouse gas emissions from deforestation and forest degradation: global land-use implications. Science, 320, 1454-1455.

Naoki, K., Duran, F. J., \& Sánchez, J. E. (2003). La avifauna de un fragmento de bosque secundario en el Valle Central, Costa Rica: su estacionalidad e implicación para la conservación. Brenesia, 59, 49-64.
Neter, J., Kutner, M. H., Nachtsheim, C. J., \& Wasserman, W. (1996). Applied linear statistical models. Boston, MA.: McGraw-Hill.

Perfecto, I., Rice, R. A., Greenberg, R., \& Van der Voort, M. E. (1996). Shade Coffee: a disappearing refuge for biodiversity. Bioscience, 46, 598-608.

Ramankutty, N., Evan, A.T., Monfreda, C., \& Foley, J.A. (2008). Farming the planet: geographic distribution of global agricultural lands in the year 2000. Global Biogeochemical Cycles, 22, GB1003.

Reitsma, R., Parrish, J. D., \& McLarney, W. (2001). The role of cacao plantations in maintaining forest avian diversity in southeastern Costa Rica. Agroforestry Systems, 53, 185-193.

Sandoval, L. (2011). Inicio de la época reproductiva y tiempo de defensa del territorio en machos de Colinus leucopogon (Galliformes: Odontophoridae). Revista de Biología Tropical, 59, 363-372.

Sandoval, L., \& Barrantes, G. (2009). Relationship between species richness of excavator birds and cavityadopters in seven tropical forests. Wilson Journal of Ornithology, 121, 75-81.

Sandoval, L., \& Sánchez, C. (2017). Lista de aves de Costa Rica: vigésima quinta actualización. San José, Costa Rica: Unión de Ornitólogos de Costa Rica.

Solano-Ugalde, A. (2011). La Avifauna del Centro de Conservación Santa Ana, San José, Costa Rica. Zeledonia, 11, 39-42.

Soulsbury, C. D., \& White, P. C. (2016). Human-wildlife interactions in urban areas: a review of conflicts, benefits and opportunities. Wildlife Research, 42, 541-553.

Stiles, F. G. (1985). Conservation of forest birds of Costa Rica: problems and perspectives. In A. W. Diamond, \& T. S. Lovejoy (Eds.), Conservation of tropical forest birds. (Technical Publication Number 4; pp. 121-138). Cambridge, UK: International Council for Bird Preservation.

Stiles, F. G. (1990). La avifauna de la Universidad de Costa Rica y sus alrededores a través de veinte años (19681989). Revista de Biología Tropical, 38, 361-381.

Stiles, F. G. (1994). A study of fall migration of Nearcticbreeding landbirds in central Costa Rica. Bird Conservation International, 4, 71-89. 
Stiles, F. G., \& Skutch, A. F. (1989). A guide to the birds of Costa Rica. Ithaca, NY: Comstock.

Stotz, D. F., Fitzpatrick, J. W., Parker, T. A. II, \& Moskovits, D. K. (1996). Neotropical birds. Ecology and Conservation. Chicago, Il: University of Chicago Press.

Tilghman, N. G. (1987). Characteristics of urban woodlands affecting breeding bird diversity and abundance. Landscape Urban Plan, 4, 481-495.

Tscharntke, T., Sekercioglu, C. H., Dietsch, T. V., Sodhi, N. S., Hoehn, P., \& Tylianakis, J. M. (2008). Landscape constraints on functional diversity of birds and insects in tropical agroecosystems. Ecology, 89, 944-951.
Vilchez Mendoza, S., Harvey, C. A., Sáenz, J. C., Casanoves, F., Carvajal, J. P., González Villalobos, J. G., ... Sinclair, F. L. (2014). Consistency in bird use of tree cover across tropical agricultural landscapes. Ecological Applications, 24, 158-168.

Weibull, A. C., Bengtsson, J., \& Nohlgren, E. (2000). Diversity of butterflies in the agricultural landscape: the role of farming system and landscape heterogeneity. Ecography, 23, 743-750.

Wunderle, J. M. Jr., \& Latta, S. C. (1996). Avian abundance in sun and shade coffee plantations and remnant pine forest in the Cordillera Central, Dominican Republic. Ornitologia Neotropical, 7, 19-34. 